
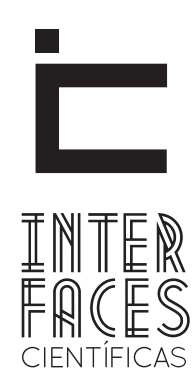

SAÚDE E AMBIENTE

\title{
A SEGURANÇA DA MOBILIZAÇ̃̃O PRECOCE EM PACIENTES CRÍTICOS: UMA REVISÃO DE LITERATURA
}

Caroline Mascarenhas Mota ${ }^{1}$

\section{RESUMO}

A imobilidade pode causar várias complicações que influenciam na recuperação de doenças críticas, incluindo atrofia e fraqueza muscular esquelética. Esse efeito pode ser reduzido com a realização de mobilização precoce no ambiente da Unidade de Terapia Intensiva (UTI), devendo ser considerados fatores neurológicos, circulatórios e respiratórios para a realização de uma mobilização precoce segura. Diante disso, este artigo tem como objetivo revisar a segurança da mobilização precoce em pacientes internados em uma UTI. Foi realizada uma revisão bibliográfica através de uma busca nas bases de dados Medline (Medical Literature Analysis and Retrieval System Online), Lilacs (Literatura Latino-Americana e do Caribe em Ciências da Saúde) e Scielo (Scientific Eletronic Library Online). A pesquisa bibliográfica resultou na obtenção de trinta e um artigos completos, dos quais foram incluídos aqueles que tinham relevância a cerca do tema em questão e selecionados cinco artigos a partir do critério de inclusão, os quais foram agrupados em uma tabela para a apresentação dos resultados. A quantidade de mobilizações realizadas encontrava-se entre 69 e 1449 sessões. A maioria dos artigos usou como critérios de mobilização os circulatórios, respiratórios e neurológicos. 0 tipo de atividade variou de movimento passivo à deambulação. Ocorreram eventos adversos em, apenas, $0,96 \%$ a $4,3 \%$ de todas as atividades realizadas. Com essa revisão foi constatada que a mobilização precoce é uma intervenção segura, que pode ser realizada em pacientes críticos internados em uma UTI.

\section{PALAVRAS-CHAVE}

Mobilização Precoce. UTI. Segurança. 


\section{ABSTRACT}

Immobility can cause several complications that influence the recovery of critical illness, including skeletal muscle atrophy and weakness. This effect can be reduced with the implementation of early mobilization in the environment of the Intensive Care Unit (ICU), and some neurological, circulatory and respiratory factors should be considered in order to have a safe early mobilization. Therefore, this article aims to review the safety of early mobilization in patients admitted to an ICU. A literature review was made through a research in Medline (Medical Literature Analysis and Retrieval System Online), Lilacs (Latin American and Caribbean Health Sciences) and SciELO (Scientific Electronic Library Online). Through the literature review, thirty-one full articles were obtained, and just the five ones which were relevant to the topic were selected. They were taken from the inclusion criteria, and were then grouped in a table in order to have the results presented. The amount of mobilizations was between 69 and 1449 sessions. Most of the items used the circulatory, respiratory or neurological mobilization criteria. The activities ranged from passive motion to walking. Adverse events occurred just in $0.96 \%$ to $4.3 \%$ of all activities. With this revision it was evident that early mobilization is a safe intervention which can be performed in critically ill patients admitted to an ICU.

\section{KEYWORDS}

Early Mobilization. ICU Security.

\section{RESUMEN}

La inmovilidad puede causar complicaciones que influyen en la recuperación de enfermedades graves, incluyendo atrofia del músculo esquelético y debilidad. Se puede reducir este efecto con la implementación de la movilización precoz en el entorno de la $\mathrm{UCl}$ (Unidad de Cuidados Intensivos), debiendo considerarse factores circulatorio, neurológico, respiratorio y llevar a cabo una movilización precoz segura. Frente a eso, este artículo tiene por objeto examinar la seguridad de la movilización precoz en pacientes ingresados en una UCI. Se realizó una revisión de la literatura a través de una búsqueda en Medline (Medical Literature Analysis and Retrieval System Online), LILACS (A Literatura Latino-Americana e do Caribe em Ciências da Saúde) y SciELO (Scientific Electronic Library Online). La búsqueda bibliográfica resultó en la obtención de los treinta y un artículos completos, entre los que se incluyeron los que tenían alguna relación con el tema en cuestión y los artículos seleccionados de los cinco criterios de inclusión, que fueron agrupados en una tabla para la presentación de los resultados. La cantidad de movilizaciones fue de entre 69 y 1.449 sesiones. La mayoría de los elementos utilizados como criterios para la movilización de los sistemas circulatorio, respiratorio o neurológico. El tipo de actividad osciló entre el movimiento pasivo para caminar. Los eventos adversos ocurrieron en el de sólo el $0,96 \%$ al $4,3 \%$ de todas las actividades. Con esta revisión se constató que la movilización precoz es una intervención segura que se puede realizar en los pacientes críticos ingresados en la $\mathrm{UCl}$.

\section{PALABRAS CLAVE}

Movilización Temprana. Unidad de Cuidados Intensivos de Seguridad.

\section{INTRODUÇ̧̃̃O}

0 repouso no leito, no passado, era frequentemente prescrito, pois se acreditava que era benéfi- co para a estabilização clínica do paciente crítico. No entanto, atualmente, sabe-se que a imobilidade 
pode influenciar na recuperação de doenças críticas, devido às alterações sistêmicas associadas a ela, como doença tromboembólica, atelectasias, úlceras de pressão, contraturas, alteração das fibras musculares de contração lenta para contração rápida, atrofia e fraqueza muscular e esquelética; além disso, pode afetar os barorreceptores, que contribuem para a hipotensão postural e taquicardia (BROWER, 2009; KORUPOLU, 2009).

Desde a década de 1940, os efeitos nocivos do repouso no leito e os benefícios da mobilização precoce têm sido reconhecidos em pacientes hospitalizados. Quando se fala em "precoce", refere-se ao conceito de que as atividades de mobilização começam imediatamente após a estabilização das alterações fisiológicas importantes, e não apenas após a liberação da ventilação mecânica ou alta da Unidade de Terapia Intensiva (UTI). A mobilização precoce na UTI pretende manter ou aumentar a força muscular e a função física do paciente. Ela inclui atividades terapêuticas progressivas, tais como exercícios de mobilidade no leito, sentado na beira do leito, em ortostase, transferência para uma poltrona e deambulação (KORUPOLU et al., 2009; NEEDHAM et al., 2009).

\section{MATERIAIS E MÉTODOS}

O presente artigo resultou de uma revisão da literatura, em que foi realizada uma pesquisa bibliográfica, através do uso das bases de dados: Medline (Medical Literature Analysis and Retrieval System Online), Lilacs (Literatura Latino-Americana e do Caribe em Ciências da Saúde) e Scielo (Scientific Eletronic Library Online). A coleta foi realizada por dois avaliadores independentes, que fizeram uma busca eletrônica de estudos entre o período de 2000-2010; artigos de anos anteriores foram utilizados de acordo com a sua importância. Durante a pesquisa, foram encontrados e utilizados artigos científicos referenciando as se-
Os benefícios da mobilização incluem melhora da função respiratória, redução dos efeitos adversos da imobilidade, melhora do nível de consciência, aumento da independência funcional, melhora da aptidão cardiovascular e aumento do bem-estar psicológico. Além disso, pode acelerar a recuperação do paciente, diminuir a duração da ventilação mecânica e o tempo de internamento hospitalar (STILLER, 2007).

Apesar dos efeitos benéficos relacionados à mobilização precoce em pacientes internados em UTI, é importante avaliar alguns fatores de segurança antes da realização dessas atividades nesse ambiente. Os principais fatores de segurança que devem ser abordados são: fatores intrínsecos ao paciente, como antecedentes médicos do paciente, reservas cardiovascular e respiratória; e fatores extrínsecos ao paciente, como acesso vascular no paciente, ambiente e equipe (STILLER, 2007).

Diante da relevância de se realizar atividades físicas durante o internamento em UTI, este artigo teve como objetivo revisar a segurança da mobilização precoce em pacientes críticos internados em UTI, a fim de esclarecer que é possível realizar este tipo de intervenção nestas unidades.

guintes palavras-chave: imobilidade, mobilização precoce, segurança, unidade de terapia intensiva.

Após o levantamento bibliográfico, realizou-se uma leitura seletiva, utilizando como critério de inclusão aqueles que faziam referência, em seus dados, a aspectos relacionados às palavras-chave anteriormente citadas e que tinham relevância a cerca do tema em questão. Foram excluídos da pesquisa os artigos sobre mobilização precoce de pacientes que não estavam internados em UTI. Posteriormente, os artigos que discutiam sobre a segu- 
rança da mobilização precoce em pacientes críticos internados em uma UTI foram agrupados em uma tabela para a apresentação dos resultados. Foram extraídos dos artigos informações sobre o tipo de estudo, composição da amostra, quantidade de mobilizações, média de idade dos participantes, critérios para mobilização, atividades realizadas e eventos adversos.

\section{RESULTADOS}

A pesquisa bibliográfica, inicialmente, resultou na obtenção de trinta e um artigos completos que estavam de acordo com as palavras-chave citadas anteriormente. Nesses artigos foram consultados seus resumos para avaliar quais realizaram intervenções em pacientes críticos internados em UTI, sendo, então, selecionados cinco artigos, os quais foram agrupados em uma tabela para a apresentação dos resultados.
Dos artigos encontrados, dois eram estudos do tipo coorte e prospectivo, dois eram estudos piloto e prospectivo e um era estudo randomizado e controlado. 0 número de pacientes que participaram das intervenções na pesquisa variou de 31 a 330, estando numa média de idade entre 54 a 68 anos. Nos cinco estudos incluídos nessa revisão observou-se que a idade não foi fator limitante para a mobilização na UTI.

Na Tabela 1 encontram-se as informações obtidas após a análise dos artigos.

\begin{tabular}{|c|c|c|c|c|c|c|c|}
\hline Autores & $\begin{array}{l}\text { Tipo de } \\
\text { Estudo }\end{array}$ & $\begin{array}{c}\mathrm{N}^{0} \mathrm{da} \\
\text { Amostra }\end{array}$ & $\begin{array}{c}\text { Quantidade } \\
\text { de } \\
\text { Mobilizações }\end{array}$ & $\begin{array}{l}\text { Média de } \\
\text { idade }\end{array}$ & $\begin{array}{c}\text { Critérios para } \\
\text { mobilização }\end{array}$ & $\begin{array}{c}\text { Tipo de } \\
\text { Atividade }\end{array}$ & $\begin{array}{l}\text { Eventos } \\
\text { Adversos }\end{array}$ \\
\hline $\begin{array}{l}\text { Kathy Stiller } \\
\text { et al,. } 2004\end{array}$ & $\begin{array}{l}\text { Estudo piloto } \\
\text { prospectivo }\end{array}$ & 31 pacientes & $\begin{array}{l}69 \\
\text { mobilizações }\end{array}$ & 57 anos & $\begin{array}{l}\text { Antecedentes } \\
\text { médicos } \\
\text { Reserva } \\
\text { cardiovascular } \\
\text { Reserva } \\
\text { respiratória } \\
\text { Fatores } \\
\text { hematológicos } \\
\text { Temperatura } \\
\text { corporal } \\
\text { Peso } \\
\text { Aparência do } \\
\text { paciente } \\
\text { Nível de } \\
\text { consciência }\end{array}$ & $\begin{array}{l}\begin{array}{l}\text { Sentar na beira } \\
\text { do leito }(56,5 \%)\end{array} \\
\text { Sentar na beira } \\
\text { da cama e de pé } \\
(27,5 \%) \\
\text { Sentar na beira } \\
\text { da cama, de pé } \\
\text { e transferência } \\
\text { para cadeira } \\
\text { (14,5\%) } \\
\text { Sentar na beira } \\
\text { da cama, de pé } \\
\text { e deambular } \\
(1,4 \%)\end{array}$ & $\begin{array}{l}\text { Alteração no } \\
\text { padrão respiratório } \\
(14,5 \%) \\
\text { Tontura }(2,9 \%) \\
\text { Queda da } \mathrm{SpO}_{2} \\
(4,3 \%)\end{array}$ \\
\hline
\end{tabular}




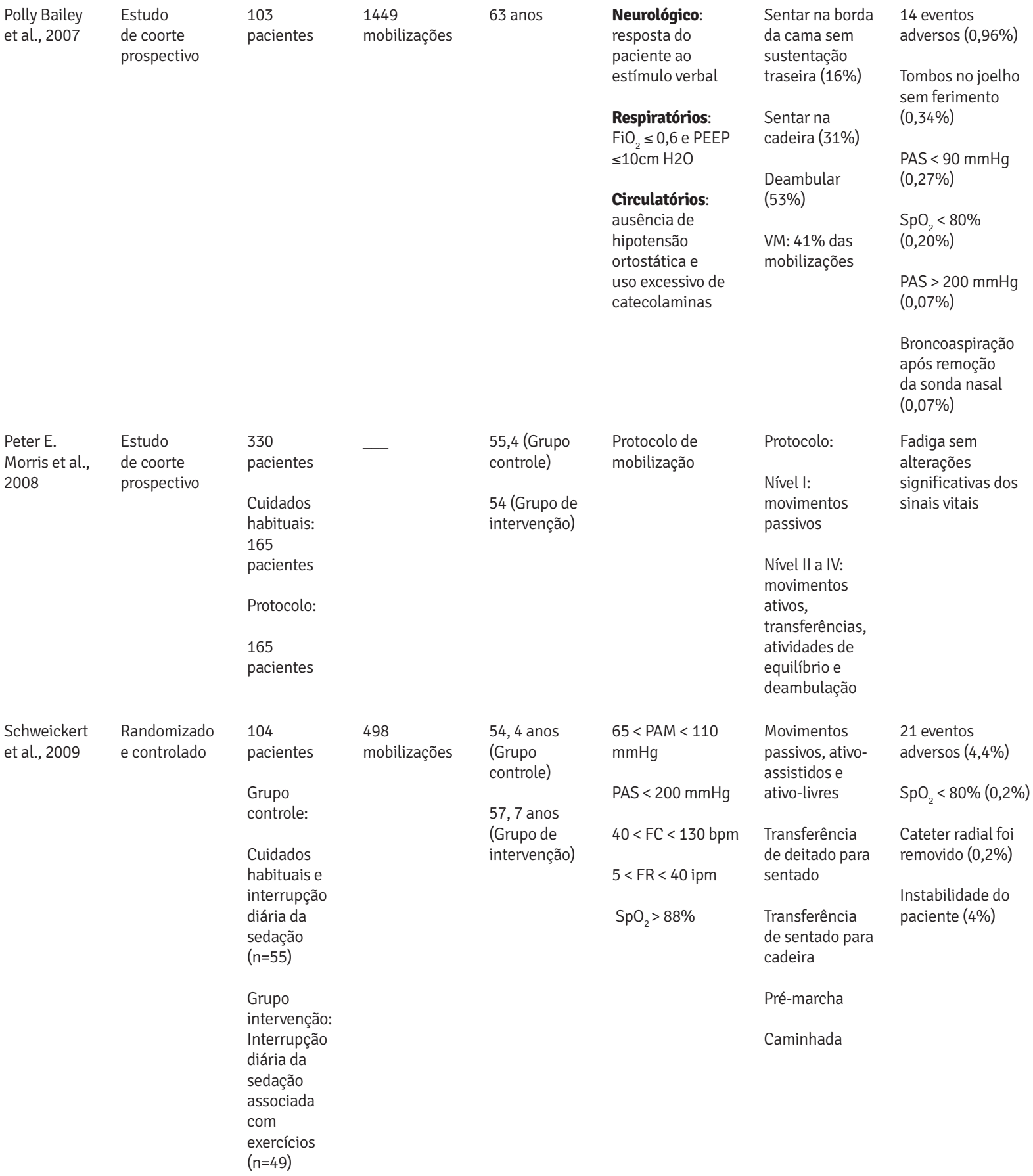


Estudo piloto 275 prospectivo

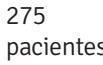

424
mobilizações

68 anos
(32-85 anos)

Avaliação médica
Avaliação da
capacidade
motora

$\begin{array}{ll}\begin{array}{l}\text { Sedestação na } \\ \text { cadeira (56\%) }\end{array} & \begin{array}{l}\text { 13 eventos } \\ \text { adversos das (3\%) }\end{array} \\ \begin{array}{ll}\text { Inclinação com } \\ \text { apoio (25\%) }\end{array} & \begin{array}{l}\text { Queda no tônus } \\ \text { muscular (1,65\%) }\end{array} \\ \begin{array}{l}\text { Inclinação sem } \\ \text { apoio (8\%) }\end{array} & \begin{array}{l}\text { Hipoxemia }\left(\mathrm{SpO}_{2}\right. \\ \leq 88 \% \text { durante } 1 \\ \text { min) }(0,94 \%)\end{array} \\ \begin{array}{l}\text { Deambulação } \\ (11 \%)\end{array} & \begin{array}{l}\text { Extubação não } \\ \text { programada } \\ (0,23 \%)\end{array} \\ & \begin{array}{l}\text { Hipotensão } \\ \text { arterial ortostática } \\ (0,23 \%)\end{array}\end{array}$

A quantidade de mobilizações realizada foi bastante diversificada, estando entre 69 a 1449 atividades realizadas. Dentre os critérios para mobilização, três das pesquisas levaram em consideração critérios neurológicos, respiratórios e circulatórios; um artigo utilizou um protocolo de mobilização (não especificado) e o outro realizou uma avaliação médica e da capacidade motora. As mobilizações encontradas variaram de movimento passivo até deambulação. E os eventos adversos ocorreram entre $0,96 \%$ a $4,3 \%$ das atividades realizadas, sendo o mais comum entre os estudos a dessaturação de oxigênio $\left(\mathrm{O}_{2}\right)$, que não foi citada apenas em um artigo, de Peter E. Morris et al. (2008), onde não houve eventos adversos.

\section{DISCUSSÃO}

Pacientes criticamente enfermos, frequentemente, permanecem em repouso no leito por vários dias ou semanas, podendo vir a sofrer complicações, tais como atrofia e fraqueza muscular e esquelética, dentre outras consequências orgânicas associadas à imobilidade (BROWER, 2009). A fraqueza muscular é cada vez mais reconhecida em pacientes admitidos em uma UTI e que sobrevivem à fase aguda de uma doença crítica. Segundo estudos realizados, ela ocorre em $25 \%$ a $60 \%$ dos pacientes que recuperam a consciência depois de uma semana de ventilação mecânica, tendo, muitas vezes, repercussões por meses ou anos após a alta hospitalar, com impacto na realização das atividades de vida diária, na qualidade de vida e reintegração do paciente à sociedade (JONGHE, 2009; VINCENT; NORRENBERG, 2009).
A mobilização precoce pode reduzir esses efeitos deletérios do repouso prolongado no leito. Burtin et al. (2009) afirmam que o exercício pode aumentar a recuperação funcional do paciente, a autopercepção do estado funcional e a força do quadríceps no momento da alta hospitalar, quando instituído precocemente em pacientes com permanência prolongada na UTI. Além disso, Kathy Stiller (2007) relata que para pacientes criticamente enfermos, a mobilização pode reduzir a incidência de complicações pulmonares, acelerar a recuperação, diminuir a duração da ventilação mecânica e do tempo de internamento hospitalar. Demonstrando-se, com isso, que tanto a saúde do paciente como a redução dos custos podem ser otimizadas com a mobilização precoce. 
Stiller e Phillips (2003) revisaram questões de segurança que devem ser consideradas quando pacientes com doença aguda forem mobilizados; os principais fatores que devem ser abordados incluem aqueles que são intrínsecos ao paciente, tais como antecedentes médicos, reservas cardíaca e respiratória; e fatores extrínsecos, como acessos vasculares no paciente, ambiente e equipe; dentre outros, que incluem fatores neurológicos e hematológicos. Segundo os autores, inicialmente, deve ser feita uma revisão sobre a história passada de disfunções cardiovascular e respiratória, medicamentos que possam afetar a mobilização e o nível funcional dos pacientes antes da internação. Em seguida, realiza-se uma investigação sobre a reserva cardiovascular, onde deve ser observada a frequência cardíaca (FC) de repouso menor que $50 \%$ da FC máxima predita para a idade, variação na pressão arterial menor que $20 \%$, eletrocardiograma sem alterações e ausência de outras doenças cardíacas. Com relação à reserva respiratória, o paciente deve apresentar uma relação $\mathrm{PaO}_{2} / \mathrm{FiO}_{2}$ maior que $300, \mathrm{SpO}_{2}$ maior que $90 \%$, padrão respiratório confortável e a ventilação deve ser continuada durante a atividade. É importante ressaltar que não é necessário que o paciente apresente todos os critérios respiratórios e circulatórios para realizar a mobilização, devendo ser levada em consideração a avaliação global do paciente e os riscos e benefícios da mobilização para o paciente.

Além desses critérios acima, destacam-se, ain$\mathrm{da}$, outros fatores que devem ser observados antes de iniciar a mobilização, tais como exames complementares, ausência de contraindicações ortopédicas e neurológicas, sinais vitais e aparência do paciente de dor, fadiga ou dispneia. A partir dessa avaliação anterior, deve-se determinar o tipo, a frequência e a intensidade das atividades. Os artigos de Kathy Stiller et al. (2004), Polly Bailey et al. (2007) e Schweickert et al. (2009), citados nesse trabalho, utilizaram critérios semelhantes. Gael Bourdin et al. (2010) utilizou como critério a avaliação da capacidade motora e avaliação médica, não sendo, no entanto, notificados quais parâmetros foram considerados; e Peter E. Morris et al. (2008) utilizou um protocolo de mobilização. Independente dos critérios utilizados para a realização da mobilização, não houve diferenças significativas nos resultados encontrados quanto à ocorrência de eventos adversos.

No estudo realizado por Polly Bailey (2007), assim como o de Peter E. Morris (2008) foi observado que o tipo de mobilização mais frequente foi deambulação, $53 \%$ e $55,1 \%$, respectivamente; contrastando com os estudos realizados por Kathy Stiller (2004) e Gael Bourdin et al. (2010), onde no primeiro a intervenção mais frequente foi sedestação na beira do leito (56,5\%) e no segundo, sedestação na poltrona (55\%). Na pesquisa de Schweickert et al. (2009) não foi notificada a frequência das mobilizações. Apesar das percentagens de mobilizações serem diferentes nos estudos, isso não foi indicativo de aumento de eventos adversos relacionados à determinada mobilização.

Foram realizadas mobilizações em pacientes mecanicamente ventilados em quatro dos artigos incluídos nesse estudo. No estudo realizado por Polly Bailey et al. (2007), 41\% das mobilizações foram realizadas em pacientes mecanicamente ventilados, das quais a deambulação foi a mais frequente; na pesquisa de Gael Bourdin et al. (2010) foi observado que 33\% dos pacientes que realizaram as atividades estavam em ventilação mecânica (VM); e 100\% dos pacientes incluídos nos estudos de Schweickert et al. (2009) e Peter E. Morris et al. (2008) encontravam-se em VM. Nesses estudos não foi observada associação entre pacientes mecanicamente ventilados e aumento de ocorrência de eventos adversos.

Os eventos adversos, descritos na literatura, relacionados à atividade são: queda sobre os joelhos, remoção de acesso vascular, pressão arterial sistólica (PAS) maior que $200 \mathrm{mmHg}$ ou menor que $90 \mathrm{mmHg}$, hipoxemia, hipotensão ortostática e extubação (BAILEY, 2007; BOURDIN, 2010). O evento adverso que apareceu na maioria dos estudos foi a dessaturação de $\mathrm{O}_{2}$, sendo ob- 
servado em 4,3\% dos eventos encontrados no estudo de Kathy Stiller et al. (2004), em 0,2\% das intercorrências observadas por Polly Bailey et al. (2007) e Schweickert et al. (2009); e no estudo de Gael Bourdin et al. (2010), onde a queda da saturação de $\mathrm{O}_{2}\left(\mathrm{SpO}_{2}\right)$ não foi quantificada. Desses estudos, foi instituído aumento do suporte de $\mathrm{O}_{2}$ nos estudos realizados por Katty Stiller et al. (2004) e Polly Bailey et al. (2007); os outros artigos não citam se houve necessidade de suplementação de $\mathrm{O}_{2}$. Esta queda da $\mathrm{SpO}_{2}$, provavelmente, ocorre devido à deficiência dos sistemas cardiorrespiratórios dos pacientes em atender ao aumento da demanda de oxigênio imposta pela mobilização (STILLER, 2004). Devendo-se destacar que esse evento não levou à interrupção das mobilizações.

Outro evento importante a se destacar foi a extubação não programada, que ocorreu em apenas um paciente mobilizado, na pesquisa realizada por Gael Bourdin (2010); no entanto, não foi preciso reintubar o paciente, devido a uma boa evolução do mesmo. Todos os eventos adversos observados foram insignificantes, não sendo critérios para interrupção da mobilização precoce.

\section{CONCLUSÃO}

Com esta revisão, foi constatado que questões de segurança devem ser consideradas quando for mobilizar pacientes críticos, tais como critérios respiratórios, circulatórios e neurológicos, pois, isso proporciona uma atividade com menor índice de eventos
A baixa incidência de problemas nesses estudos sugere que a mobilização precoce é uma atividade segura. É importante ter uma visão geral das questões de segurança antes de iniciar a mobilização, pois, isso permite observar quais pacientes estão propensos a sofrer intercorrências, além de ajudar a identificar quais sistemas estão mais susceptíveis à ocorrência de instabilidades (SCHWEICKERT et al., 2009).

Deve-se destacar, também, que um trabalho em equipe tem sido associado a uma melhor evolução do paciente. $O$ artigo de Polly Bailey et al. (2007) relata que cada evento teve a participação do fisioterapeuta, do terapeuta respiratório, do enfermeiro e do técnico de cuidado intensivo. No protocolo realizado por Morris et al. (2008) houve a participação de uma equipe de mobilidade que continha enfermeira de cuidados intensivos, auxiliar de enfermagem e fisioterapeuta. Ratificando esses estudos, Perme e Chandrashekar (2009) afirmaram que a abordagem multidisciplinar é essencial para a evolução do paciente, além de garantir segurança durante a implementação do programa de mobilização precoce. adversos. A partir da análise dos artigos foi observado que é possível mobilizar o paciente de forma segura e sem intercorrências graves. Todavia, existe uma necessidade de se realizar mais estudos acerca do tema abordado.

\section{REFERÊNCIAS}

BAILEY, P. P. et al. Culture of early mobility in mechanically ventilated patients. Critical Care Medicine, Salt Lake City, v. 37, n. 10, p. 429-435, 2009.
BAILEY, P. et al. Early activity is feasible and safe in respiratory failure patients. Critical Care Medicine, Provo, v. 35, n. 1, p. 139-145, 2007. 
BOURDIN, G. et al. The feasibility of early physical activity in intensive care unit patients: A prospective observational one-center study. Respiratory Care, Lyon, v. 55, n. 4, p. 400-407, 2010.

BROWER, R.G. Consequences of bed rest. Critical Care Medicine, Baltimore, v. 37, n. 10, p. 422-428, 2009.

BURTIN, C. et al. Early exercise in critically ill patients enhances short-term functional recovery. Critical Care Medicine, Leuven, v. 37, n. 9, p. 2499-2505, 2009.

JONGHE, B. Intensive care unit-acquired weakness: Risk factors and prevention. Critical Care Medicine, Garches, v. 37, n. 10, p. 309-315, 2009.

KORUPOLU, R.; GIFFORD, J.M.; NEEDHAM, D. Early Mobilization of Critically Ill Patients: Reducing Neuromuscular Complications After Intensive Care. Contemporary Critical Care, Baltimore, v. 6, n. 9, 2009.

MORRIS, P. E. et al. Early intensive care unit mobility therapy in the treatment of acute respiratory failure. Critical Care Medicine, Winston Salem, v. 36, n. 8, p. 2238-2243, 2008.

NEEDHAM, D.M.; TRUONG, A.D.; FAN, E. Technology to enhance physical rehabilitation of critically ill patients. Critical Care Medicine, Baltimore, v. 37, n. 10, p. 436-441, 2009.
PERME, C.; CHANDRASHEKAR, R. Early mobility and walking program for patients in intensive care units: creating a standard of care. American Journal of Critical Care, Columbia, v. 18, p. 212-221, 2009.

SCHWEICKERT, W.D. et al. Early physical and occupational therapy in mechanically ventilated, critically ill patients: a randomised controlled trial. The Lancet, Chicago, v. 373, p. 1874-1882, 2009.

STILLER, K. Safety issues that should be considered when mobilizing critically ill patients. Critical Care Clinics, Adelaide, v. 23, p. 35-53, 2007.

STILLER, K.; PHILLIPS, A. Safety aspects of mobilising acutely ill inpatients. Physiotherapy Theory and Practice, Adelaide, v. 19, p. 239-257, 2003.

STILLER, K.; PHILLIPS, A. C.; LAMBERT, P. The safety of mobilisation and its effect on haemodynamic and respiratory status of intensive care patients. Physiotherapy Theory and Practice, Adelaide, v. 20, p. 175185, 2004.

VINCENT, J.L.; NORRENBERG, M. Intensive care unitacquired weakness: Framing the topic. Critical Care Medicine, Brussels, v. 37, n. 10, p. 296-298, 2009. 